
THU0339 IMPACT OF CAROTID ULTRASOUND ON THE CARDIOVASCULAR RISK STRATIFICATION OF PATIENTS WITH SYSTEMIC LUPUS ERYTHEMATOSUS

J.C. Quevedo Abeledo ${ }^{1},{ }^{2} \mathrm{H}$. Sánchez, I. Rua-Figueroa ${ }^{1}$, B. Tejera ${ }^{3}$, A. Naranjo ${ }^{1}$, C. Rodríguez-Lozano ${ }^{1}$, I. Ferraz-Amaro ${ }^{2} .{ }^{1}$ Rheumatology Division, Hospital Universitario de Gran Canaria Dr Negrín, Las Palmas de Gran Canaria; ${ }^{2}$ Rheumatology Division, Hospital Universitario de Canarias, Tenerife; ${ }^{3}$ Rheumatology Division, Complejo Hospitalario Universitario Insular, Las Palmas de Gran Canaria, Spain

Background: Autoimmune rheumatic diseases, including systemic lupus erythematosus (SLE), are associated with a significant increase in cardiovascular morbidity and mortality. The risk stratification instruments used in the general population underestimate the true risk of events in these patients. Carotid ultrasonography, through the detection of subclinical atheromatosis, is a powerful predictor of future cardiovascular events. The available evidence, endorsed in the official Prevention Guidelines, supports the use of this technique for the adequate identification of those patients of "very high risk", candidates for preventive interventions of greater intensity.

Objectives: To analyse the cardiovascular risk profile, the prevalence of subclinical atheromatosis detected by carotid echography, and its implications in the prevention strategy in patients with SLE.

Methods: A cross-sectional study of 276 patients diagnosed with SLE. The clinical characteristics and risk profile were analysed by SCORE. The presence of plaques and intima-media thickness (cIMT) was determined by carotid ultrasound and the cIMT percentiles were calculated according to tables adjusted for age and sex. Differences in risk stratification before and after carotid ultrasonography were determined by univariate regression analysis. The therapeutic implications after reclassification were evaluated according to the 2016 European Prevention Guides.

Results: Risk stratification using SCORE was: low in 187 (67\%), moderate in 73 $(26 \%)$, and high or very high in $16(6 \%)$. The median percentiles for cIMT were not statistically different from the $p 50$ of healthy general population $(p=0.54)$. Ultrasound showed the presence of plaque or cIMT $>$ p75 or cIMT $>0.90 \mathrm{~mm}$ in $60 \%$ (166) of the patients evaluated. The presence of this finding by risk categories was: low 102/187 (55\%), moderate 55/73 (75\%), high 7/9 (78\%) and very high 6/7 $(86 \%)$. According to the published guidelines, the detection of plaque carried out the re-stratification to a very high risk in $35 \%$ of patients. These patients where reclassification was achieved showed a higher SLICC index compared to those that did not change of category (mean difference 0.9 points, $p=0.000$ ). This difference remained statistically significant when the items related to cardiovascular risk that SLICC possesses were eliminated. SLEDAI and Katz were not statistically different between both groups. The SCORE of patients who were reclassified was also statistically higher (mean difference 0.7 points, $p=0.021$ ). Ninety percent of patients were re-classified to a very high risk had out-of-target LDL cholesterol levels for their new risk category ( $\mathrm{LLL}<70 \mathrm{mg} / \mathrm{dl}$ ). Similarly, only $46 \%$ of them were on statin therapy. Therefore, the indication or intensification of lipid-lowering treatment was followed only in $30 \%$ of the total evaluated.

Conclusions: The reclassification of cardiovascular risk through the use of ultrasonography in SLE occurs in one third of patients. Our data suggests that this may be consequence of the damage produced by the disease. One in three candidates needed preventive interventions of greater intensity that had not been taken.

Disclosure of Interest: None declared

DOI: 10.1136/annrheumdis-2018-eular.5996

\section{THU0340 PROLONGED REMISSION IS ASSOCIATED WITH A REDUCED RISK OF CARDIOVASCULAR DISEASE IN PATIENTS WITH SYSTEMIC LUPUS ERYTHEMATOSUS}

${ }^{1}$ S. Fasano, D.P. Margiotta ${ }^{2}$, L. Pierro ${ }^{1}$, A. Riccardi ${ }^{1}$, A. Afeltra ${ }^{2}$, G. valentini ${ }^{1}$ ${ }^{1}$ Rheumatology unit, Università degli studi della Campania Luigi Vanvitelli, Naples; ${ }^{2}$ Clinical Medicine and Rheumatology Department, Campus Bio-Medico University of Rome,, Rome, Italy

Background: Cardiovascular disease (CVD) has emerged as one of the most important causes of mortality in systemic lupus erythematosus (SLE) ${ }^{1}$. In previous studies, disease activity, as assessed by SLEDAI (at the first visit or as mean annual value registered during follow-up), did not result to have any predictive role on the subsequent occurrence of $C V D^{2-3}$.

Objectives: To investigate the relationship between prolonged remission and the occurrence of a subsequent first CV event in patients with SLE.

Methods: Out of 452 patients consecutively admitted to two tertiary Italian centres from November 1st 2000 to December 31st 2016, the 409 patients, who, at admission, had not experienced any CV event, had not received any anticoagulation therapy and had been visited at least biannually during follow-up, were considered for the present study. Prolonged remission was defined as a 5 year consecutive period of no disease activity based on SLEDAI-2K ${ }^{3}$.. Patients with prolonged remission were furtherly subdivided according to Zen et al ${ }^{4}$ into 3 groups: complete remission, clinical off-corticosteroids remission (offCR), clinical on-corticosteroids remission (onCR). Kaplan-Meier curves and the log-rank test were used to analyse differences in event-free survival between groups. Cox regression analysis was used to investigate disease and therapeutic features associated with the development of a first CV event.

Results: During 72 months median follow-up time, 29 (7.0\%) CV events occurred (two events in patients who had undergone prolonged remission). Out of the 409 patients, 28 patients $(6.8 \%)$ achieved a prolonged complete remission, $13(3.1 \%)$ prolonged clinical offCR and 64 (15.5\%) prolonged clinical onCR. Kaplan-Meier analysis revealed a greater overall $\mathrm{CV}$ event-free rate in patients achieving a prolonged remission compared to those in remission but for less than 5 years and patients not in remission (logrank test $\chi 2=19.82 ; p=0.0001$; figure 1). However, at Kaplan-Meier analysis, CV outcome was similar among patients in prolonged remission, irrespective of the type of remission achieved $(p>0.05)$. At multivariate analysis, treatment with hydroxychloroquine for more than 5 years and prolonged remission were protective (HR 0.38; $95 \% \mathrm{Cl} 0.16-0.90$; $\mathrm{HR} 0.08,95 \% \mathrm{Cl} 0.01-$ 0.53 ) while antiphospholipid syndrome increased the risk of a first CV event (HR $3.80 ; 95 \% \mathrm{Cl} 1.68-8.61$ ). No differences were found between patients treated or not with aspirin. Nevertheless, among patients from Rome cohort, aspirin was only prescribed to patients with high traditional $\mathrm{CV}$ risk score.

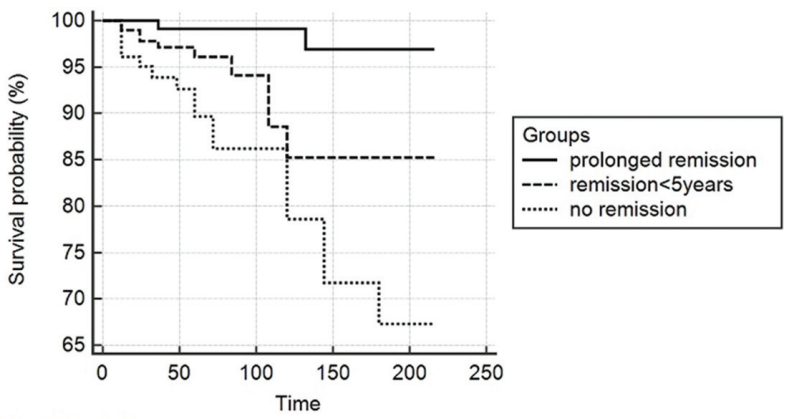

Number at risk

Group: prolonged remission

$\begin{array}{cccccc}119 & 107 & 68 & 29 & 17 & 0\end{array}$

$\begin{array}{lllllll}187 & 92 & 34 & 15 & 4 & 0\end{array}$

$\begin{array}{cccccc}\text { Group: no remission } & & & & & \\ 103 & 62 & 36 & 19 & 13 & 0\end{array}$

Conclusions: A prolonged remission, whichever the subtype, is associated with a better CV outcome and should be considered as a treat-to-target goal in the CV risk management of the lupus patient.

\section{REFERENCES}

[1] Cervera R, et al. Medicine (Baltimore) 2003.

[2] ludici M, et al. Rheumatology (Oxford) 2016.

[3] Fasano S, et al. Lupus 2017

[4] Zen M, et al. Ann Rheum Dis 2017.

Disclosure of Interest: None declared

DOI: 10.1136/annrheumdis-2018-eular.3222

\section{THU0341 LOW VITAMIN D IS ASSOCIATED WITH THROMBOSIS IN SYSTEMIC LUPUS ERYTHEMATOSUS}

M. Petri, W. Fu, D.W. Goldman. Medicine, Johns Hopkins University School of Medicine, Baltimore, USA

Background: Low vitamin $D$ is common in systemic lupus erythematosus (SLE). It is also found in antiphospholipid syndrome. Vitamin D has effects on tissue factor, PAl-1, thrombomodulin and platelet aggregation that suggest it has an antithrombotic role. We asked whether low vitamin D was associated with thrombosis in SLE, adjusting for lupus anticoagulant.

Objectives: We asked whether low vitamin D was associated with thrombosis in SLE, adjusting for lupus anticoagulant.

Methods: A total of 1,392 SLE patients were included in the analysis. At the first visit when vitamin D was measured, $76.7 \%$ had levels of 25 -hydroxyvitamin $\mathrm{D}<40 \mathrm{ng} / \mathrm{mL}$. The SLE patients were: $92 \%$ female, mean age 42.9 years, and ethnicity $50 \%$ Caucasian, $41 \%$ African American. $27 \%$ patients had a history of thrombosis; $7 \%$ stroke, $4 \% \mathrm{Ml}$ and $14 \% \mathrm{DVT}$.

Results: Vitamin D, measured either as a continuous variable or as "low" $(<40 \mathrm{ng} / \mathrm{mL})$ vs. normal, was associated with any thrombosis and with DVT. 